\title{
DFT studies of the refractive index of boron-doped diamond
}

\author{
B. Dec, R. Bogdanowicz \\ Faculty of Electronics, Telecommunications and Informatics, Gdańsk University of Technology, G. Narutowicza \\ 11/12, 80-233 Gdańsk
}

Received June 09, 2018; accepted June 19, 2018; published June 30, 2018

\begin{abstract}
The density functional theory is one of the optimal solutions in calculation of optical properties of materials on the quantum scale. In this paper, we have investigated the refractive index of a boron-doped diamond structure with the usage of Atomistic Toolkit software from Synopsys. During this study, various methods and pseudopotentials were checked to obtain an optimal performanceaccuracy method for calculation of such materials. The leading method used in calculation was meta-GGA with Fritz-Haber Institute pseudopotential.
\end{abstract}

The Density Functional Theory (DFT) [1] every year gains more popularity in the scientific community for being the most cost-effective solution for calculating electronic properties of materials on the quantum scale. Practical usage of DFT is based on approximations of the exchange-correlation potential. This potential describes the effects of the Pauli principle on the many-electron system. Moreover, general gradient approximation with Perdew, Burke, and Ernzerhof (PBE) [2] exchangecorrelation energy was chosen as state of the art approach in DFT calculations.

Electronic properties of BDD have been studied over years in the literature [3]. Most of the work has been done with an error of $20-30 \%$. Optical characteristics have not been so widely studied because of the lack of accuracy. The actual state of the art in DFT gives high precision to the study of optical properties of materials in a virtual environment. The Kubo-Greenwood formula was used to calculate the susceptibility tensor shown in the work of Desjarlais [4], which is related to the response coefficients, relative dielectric constant $\epsilon_{\mathrm{r}}$, polarizability $\alpha$, and optical conductivity $\sigma$.

DFT [1] with the Linear Combination of Atomic Orbitals (LCAO) [5] method was applied to the calculation of geometry relaxation and optical properties of complex structures with large numbers of atoms. In those calculations, we have used, developed by Soler et al. [6], a fully self-consistent DFT based on a linear combination of atomic orbitals. Exchange and correlation are treated within Kohn-Sham DFT [6]. We have also used standard norm-conserving pseudopotentials [7] in their fully nonlocal form.
Calculations were done in a flexible, efficient and innovative calculation package: Atomistic Toolkit from Synopsys [8] that uses Siesta implementation of DFT routines. The meta-GGA calculator was chosen from a variety of available options [9]. Moreover, a few pseudopotentials were checked as the most accurate in calculation that can be obtained using DFT method.

The slab was created with 8 atoms (see Fig. 1.) as a supercell using Face Centered Cubic (FCC) lattice with cell dimensions of $a=3.56679 \AA$ [10]. Special firstprinciple norm-conserving pseudopotential Fritz-Haber Institute [11], with the Monkhorst-Pack [12] scheme for Brillouin zone was applied for k-point sampling.

In principle, we verify if meta-GGA functional could contribute a relatively high accuracy of simulations of a boron-doped diamond structure as well as allow critical correction of experimental results in this matter. a)

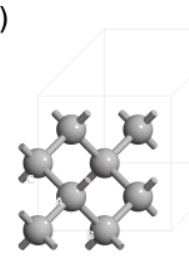

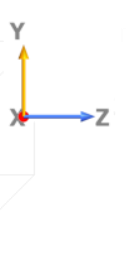

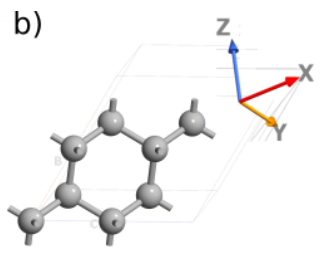

Fig. 1. Diamond structure slabs used in calculations. On a) we can see lattice in $\mathrm{Y}, \mathrm{Z}$ - direction, in $\mathrm{b}$ ) we can see in a $3 \mathrm{D}$ diamond structure.

In the procedure of calculation method selection, the accuracy of band structure determination was chosen as the major measure of simulation correctness. The accuracy of bandgap estimation was compared and evaluated with the help of experimental data [13-14]. Calculation results were also compared with the work of Maezono [15], who used the Monte Carlo method, which is the most accurate approach known in the literature. The main drawback of the Monte Carlo method is the cost of computational resources, which limits, in practice, the structures sizes to 50 atoms. 
Such a low number of atoms allowed for creating scripts that compare the results of different methods and pseudopotentials with different basis sets. For practical usage, we consider the evaluating time of results and accuracy of chosen models. Chosen models will be used to calculate the refractive index in order to verify that the assumptions concerning the methods used and pseudopotentials are correct.

Results were obtained in a bandgap of $5.11426 \mathrm{eV}$ for the indirect bandgap in a diamond and $6.61637 \mathrm{eV}$ for the direct bandgap. In comparison to the experimental data, the error was $6.5 \%$ for the indirect bandgap [5] and $9.4 \%$ for the direct bandgap. Such an error is acceptable and the models and thus the approach could be used to simulate much more complex structures with high accuracy. The refractive index was derived using the approach validated during basic calculations (see Fig. 2).

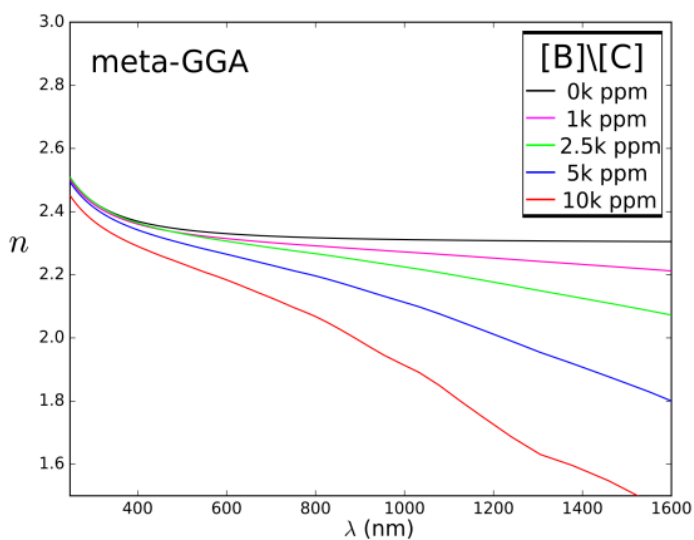

Fig. 2. Refractive indices of boron- doped diamond with dopant levels from $0 \mathrm{k}$ ppm to $10 \mathrm{k}$ ppm $[\mathrm{B}] \backslash[\mathrm{C}]$.

The refractive index is one of the most important parameters that is needed for the design sensors, waveguides or fiber coatings. Next, it defines the coupling parameters of different materials and means that with increasing the refractive index we obtain higher optical density.

The obtained results indicate that changes in the characteristics of the refractive index are in good agreement with the experiments done by Sobaszek et al. [13] The calculated values of a bulk diamond were 2.51-2.31 for a wavelength extending from $200 \mathrm{~nm}$ up to $1600 \mathrm{~nm}$. The measured values were $2.55-3.37$ for a wavelength in the range from $200 \mathrm{~nm}$ up to $1600 \mathrm{~nm}$. This indicates that the error obtained for bulk diamond is less than $2.5 \%$. For doped diamonds, we have obtained the results with a similar error which does not exceed $5.4 \%$. The full set of results of DFT calculations is listed in Table 1. Experimental data from [13] were shown for comparison.
Tab. 1. Refractive index calculated and measured [13] at $550 \mathrm{~nm}$ of a boron-doped diamond with an increasing doping level.

\begin{tabular}{|l|l|l|l|}
\hline$[\mathrm{B}] /[\mathrm{C}]$ & Meta-GGA & Experiment $[14]$ & Error \\
\hline $0 \mathrm{k} \mathrm{ppm}$ & 2.35 & 2.45 & $4.1 \%$ \\
\hline $1 \mathrm{k} \mathrm{ppm}$ & 2.31 & 2.40 & $3.3 \%$ \\
\hline $2.5 \mathrm{k} \mathrm{ppm}$ & 2.30 & 2.43 & $5.4 \%$ \\
\hline $5 \mathrm{k} \mathrm{ppm}$ & 2.27 & 2.36 & $3.8 \%$ \\
\hline $10 \mathrm{k} \mathrm{ppm}$ & 2.21 & 2.2 & $0.5 \%$ \\
\hline
\end{tabular}

The results obtained by the use of the meta-GGA method with FHI pseudopotentials are in close agreement with experiments [13-14], [16-18]. We can compare them to hybrid Becke three-parameter Lee-Yang-Parr (B3LYP) exchange functional [19], which is much more costly computationally. The use of a meta-GGA method allows us to obtain a bandgap of $5.61 \mathrm{eV}$. The use of B3LYP results in a less accurate bandgap with a value of $5.66 \mathrm{eV}$ [20]. It should be noticed that simulated and experimental refractive indices show high agreement despite ignoring crystal borders in simulated materials.

As the main conclusion, it is safe to claim that a metaGGA method is accurate enough to calculate the optical properties of doped diamond structures and allows to predict diamond properties before application in optical systems.

The authors gratefully acknowledge the financial support from the Polish National Science Centre (NCN) under Grant No. 2016/21/B/ST7/01430, 2016/22/E/ST7/ 00102, 2014/14/M/ST5/00715 and the National Centre for Science and Development Grant Techmatstrateg No. 347324 2015/16/T/ST7/00469. This work was partially supported by the Science for Peace Programme of NATO (Grant no. G5147). The DS funds of the Faculty of Electronics, Telecommunications, and Informatics of the Gdansk University of Technology are also acknowledged.

\section{References}

[1] W. Kohn, L.J. Sham, Phys. Rev. 40(4) A1133 (1965).

[2] J.P. Perdew, K. Burke, M. Ernzerhof, Phys. Rev. Lett. 77(18), 3865 (1996).

[3] Y. Yan, J. Gong, Z. Zong, Thin Solid Films 518(17), 4989 (2010).

[4] M.P. Desjarlais, Contrib. Plasma Phys. 45(3-4), 300 (2005).

[5] L.G. Ferreira, M. Marques, L.K. Teles, Phys. Rev. B 78(12), 125116 (2008).

[6] J.M. Soler et al., J. Phys.: Cond. Matter 14(11), 2745 (2002).

[7] L. Kleinman, D. Bylander, Phys. Rev. Lett. 48(20), 1425 (1982).

[8] Synopsys QuantumWise, Atomistix Toolkit version 2017.12.

[9] F. Tran, P. Blaha, Phys. Rev. Lett. 102(22), 226401 (2009).

[10] D.R. Lide, Ed., CRC Handbook of Chemistry and Physics, 83rd Edition (CRC Press 2002).

[11] N. Troullier, J.L. Martins, Phys. Rev. B 43(3), 1993 (1991). 
[12] W.A. Harrison, Solid state theory (McGraw-Hill, 1970).

[13] M. Sobaszek et al., Opt. Materials 42, 24 (2015).

[14] M. Ficek et al., Appl. Surface Science 387, 846 (2016)

[15] R. Maezono, A. Ma, M.D. Towler, R.J. Needs, Phys. Rev. Lett. 98(2), 025701 (2007)

[16] R. Bogdanowicz et al., J. Opt. Soc. Korea, JOSK 19(6), 705 (2015).

[17] M. Ficek et al., Opt. Mater. Expr. 7(11), 3952 (2017).

[18] M. Ficek, R. Bogdanowicz, J. Ryl, Acta Phys. Polon. A 127(3), 868 (2015).

[19] P.J. Stephens, F.J. Devlin, C.F. Chabalowski, M.J. Frisch, J. Phys. Chem. 98(45), 11623 (1994).

[20] P. Rivero, W. Shelton, V. Meunier, Carbon 110, 469 (2016). 